\title{
Are we meeting current standards in medicines reconciliation? A study in a District General Hospital.
}

\author{
Emma Iddles, Andrew Williamson, Alison Bradley, Khurram Khan
}

Hairmyres Hospital, NHS Lanarkshire

\begin{abstract}
Introduction: Inadequate medicines reconciliation on admission is often identified as a major cause of patient morbidity, with poor access to patient's regular medications often cited as a barrier to care. In the surgical admission unit of our district general hospital, drug charts are completed by junior doctors who do not have access to the Emergency Care Summary (ECS) thus making it difficult to accurately complete admission drug charts.

Methods: Our initial measurement of all acute surgical admissions revealed that $49 \%$ of patients had an accurate medicines reconciliation upon admission, increasing to $75 \%$ within 24 hours of admission. It was clear from this data that our current practice needed improvement in order to ensure patient safety. Resultantly the junior medical staff were provided with ECS accounts and teaching to aid the process of medicines reconciliation.
\end{abstract}

Results: Following the introduction of access to ECS and junior doctor education, a further two data cycles were completed. On the first cycle, the number of accurately completed drug charts increased to $62 \%$ on admission and $86 \%$ at 24 hours. After the second cycle $57 \%$ were complete on admission increasing to $84 \%$ at 24 hours.

Conclusion: Our project has shown that by providing junior doctors with medicines reconciliation education and access to patients' preadmission medications through a nationwide electronic system resulted in a considerable increase in the completion of medicine reconciliation.

\section{Problem}

Medicines reconciliation is an important part of the admissions process whereby medical staff identify patient's regular and acute medications, drug allergies, and over the counter prescriptions. A routine audit investigating medicine reconciliation in a busy district hospital in East Kilbride, Glasgow revealed that this was poorly executed on admission. This was a particular problem within the acute surgical receiving wards. A previous audit carried out by the pharmacy department showed that the department was failing to meet current National Institute for Clinical Excellence (NICE) guidelines which states a $95 \%$ standard that medicines reconciliation should be carried out within 24 hours of admission.

This issue was generated because the admitting doctors were unable to complete medicine reconciliation at time of admission because the junior medical staff did not have access to their regular medications on the Emergency Care Summary (ECS). ECS is an online summary of patient demographics that can be accessed by primary and secondary care. Generally, medicine reconciliation was completed during the patient's admission by the ward pharmacists. However, it was noted that patients frequently did not have their ECS accessed or drug chart completed within 24 hours of admission. This was mainly a problem occurring out of hours and during the weekend as doctors did not have the additional support of pharmacists. This presented a compromise to patient care as regular medications were often omitted from the drug chart.

\section{Background}

Medication errors at the interface of care (admission, transfer, and discharge) are the leading cause of patient morbidity and mortality [1]. Medicine reconciliation aims to enhance patient safety by providing an accurate record of the patient's medication before and during admission. This information is commonly collated by junior medical staff and pharmacists and includes medication, dosage, frequency, and allergy status. Medicines reconciliation may need to be carried out multiple times during the patient's admission as acute medications and procedures may require a change of regular medications. Factors affecting poor medicines reconciliation include no access to patient's regular prescription, out of hours prescribing and the time constraints of busy wards. Guidelines published by NICE and Scottish Patient Safety Programme (SPSP) have highlighted the need for accurate medicine reconciliation [2]. It sets a $95 \%$ standard that all medicines should be reconciled within 24 hours of the patient's admission.

Poor medicines reconciliation has been noted to be a significant problem across several health boards. Several past projects have sought to improve this, introducing interventions with varying degrees of success. Hughes et al. demonstrated a moderate improvement of $60 \%$ to $85 \%$ in all surgical admissions following introduction of a "medicines on admission" proforma [3]. Burnswicker and Yogarajah had less success in the preassessment clinics of a General surgical and Urology department [4]. Despite measures including policy changes to such as completion of all 
drug charts at the end of the clinics, in addition to supportive measures including education, email reminders, and posters, they only managed a completion rate of $51 \%$. Whilst both of these projects do show small interventions can increase overall medicines reconciliation, the fact that both ultimately fall short of the $95 \%$ target highlights further changes are needed.

\section{Baseline measurement}

Baseline data was collected in a busy acute surgical receiving ward. During this collection period the junior medical staff did not readily have access to ECS.

All surgical admissions admitted during a one week period in November 2014 had their medical notes and drug chart reviewed. Data collected included patient details, time and date of admission, and whether medicines reconciliation was completed on admission and at 24 hours of hospital admission. 74 data sets were collected. Of these, six were lost to follow up and three were discharged within 24 hours of admission. $46 \%$ of surgical admissions had an accurate reconciliation of their medications on admission and $77 \%$ within 24 hours of admission.

\section{Design}

From the baseline data it was clear that medicines reconciliation was not being carried out effectively within 24 hours of patient admission. The main factors in determining the quality of information gathering included access to ECS, admitting patients out of hours and time constraints due to volume of admissions and reduced staffing numbers.

It was appreciated that that some factors were more difficult to modify so we focused on improving education regarding medicines reconciliation and access to ECS for junior medical staff.

\section{Strategy}

PDSA 1: A previous audit carried out by the pharmacy department showed that $0 \%$ of patients had completed medicine reconciliation within 24 hours of admission.

PDSA 2: Data was collected over a one week period, entered into a database and analysed. During this initial cycle we learnt that it was difficult to follow up all patients admitted into the ward due to the rapid turnover. We endeavoured to improve this by collecting data at multiple points during the day and consulting the ward ' 24 hour' book which keeps a record of all discharges and transfers from the ward.

PDSA 3: The junior medical staff were educated about the importance of medicines reconciliation and current NICE guidelines during a lunchtime teaching session. The importance of this information was reinforced through email. This was in addition to formal teaching delivered by the pharmacy department regarding medicines reconciliation at the FY1 induction.
PDSA 4: Access to ECS was successfully gained for junior medical staff working in the surgical department. They were given log in details and instruction of use of the program.

PDSA 5: A second data collection was performed. Results were compiled into a database.

PDSA 6: A final data collection was made and analysed.

\section{Post-measurement}

The main outcome measured was that medicine reconciliation was fully completed within 24 hours of the patient's admission. We also measured the percentage of patients who had this information completed on admission.

A previous audit within the surgical department showed that $0 \%$ of patients had a completed medicines reconciliation within 24 hours of admission.

Initial results demonstrated that $46 \%$ of patients had medicine reconciliation completed on admission and $77 \%$ within 24 hours of admission.

Measures were put in place to help improve medicines reconciliation, including improving access to ECS and education of junior medical staff.

The second data collection showed that $62 \%$ of patients on admission and $86 \%$ at 24 hours had a fully completed drug chart whilst $93 \%$ had an accurate medicine history recorded.

The third cycle of data collection showed that $57 \%$ and $84 \%$ of patients had a completed drug chart on admission and at 24 hours respectively and $89 \%$ had a complete medication history recorded.

The initial data set showed that the surgical receiving ward was under performing. Once this had been highlighted to the stake holders there was marked increase in the completed medication reconciliation occurring at both admission and within 24 hours. However, this number begins to fall shown by the third data collection. This highlights the need for continual education about the importance of medicines reconciliation among junior staff. A variety of factors could affect these figures. The main factor being medical staff. Several junior doctors rotate throughout the department weekly. New doctors working on the ward may not have received the same education as previous staff. The receiving department is also very busy and time constraints may result in a less accurate medications history being performed.

We propose that formal medicines reconciliation training for all junior medical staff is mandatory. Also, ensuring that those involved in medicines reconciliation have full access to ECS will help improve standards. Continual reeducation of staff is important to maintain these standards.

See supplementary file: ds5278.jpg - "Figure 1: Graph of Medicines Reconciliation Targets Achieved" 


\section{BMJ Quality Improvement Reports}

\section{Lessons and limitations}

As this project was conducted largely by junior doctors, there are a number of valuable lessons we have learned:

1. Due to our commitments to ward work it was often difficult to make time to collect data throughout the day. This often resulted in data being collected near the end of our shifts rather than directly on admission. As a result, several patients, particularly during the baseline measurement, were lost to follow up.

2. The project has highlighted the importance of communication amongst colleagues of various grades and specialties when reconciling patient medication, as involvement of junior and middle grade medical staff and pharmacists were vital to improvement.

3. The project only looked at completion of charts on admission and within 24 hours with no investigation into how this affected patient care. It may have been useful to investigate if the improvements made had any effect on inpatient outcomes and discharge.

4. Finally, the marked increase in number of charts completed within 24 hours highlights how a simple intervention, in this instance providing ECS access, can have a dramatic increase in the quality of patient care. However, the lower instance of drug charts complete on admission, and the drop in completion rates in 24 hours during the second repeat cycle shows that further intervention is needed in both increasing reconciliation on admission and maintaining improvement in this area long term.

\section{Conclusion}

From our results it is clear that having access to ECS amongst junior medical staff can lead to a significant increase in accurate medicines reconciliation. Following introduction of ECS, the number of drug charts completed within 24 hours increased from our baseline measurement of $75 \%$ to $86 \%$ after our first cycle and $84 \%$ after the second. However, given that both our results still fall short of the $95 \%$ target as recommended by the NICE guidelines, and that our results slightly decreased between repeat cycles, it is clear that further intervention is needed to bring our practice into line with current recommendations and to maintain our improvements over a long term basis.

\section{References}

1. Vira, T, Colquhoun, M and Etchells, E. Reconcilable differences: correcting medication errors athospital admission and discharge. Qual Saf Health Care 2006;15:122-126. doi: 10 .

2. National Institute for Clinical Excellence (NICE). Technical patient safety solutions for medicines reconciliation on admission of adults to hospital, 2007.

3. Hughes E, Hegarty P, Mahon A. Improving Medication Reconciliation on the Surgical Wards of a District General HospitalBMJ Qual Improv Report 2012;1: doi:10.
4. Brunswicker A, Yogarajah A. Improving pre-operative medicines reconciliation. BMJ Quality Improvement Reports 2014; u205475.w2230 doi: 10.

\section{Declaration of interests}

Nothing to declare.

\section{Acknowledgements}

We would like to thank Consultant surgeon Alistair Brown for providing ECS, Surgical pharmacists Lisa and Karen Cashin for helping with ECS distribution and providing access to previous pharmacy audits, and for the surgical FY1s of Hairmyres Hospital for engaging with the intervention.

\section{Ethical approval}

After Consulting the West of Scotland Research Ethics service guidelines we have not applied for ethical approval as it does not meet requirements. 